\title{
Propuestas para \\ "democratizar" los beneficios de los biobancos en América Latina
}

Claude Vergès'

Universidad de Panamá

\author{
María Angélica Sotomayor Saavedra ${ }^{2}$ \\ Universidad de Chile
}

Patricia Sorokin ${ }^{3}$

Universidad de Buenos Aires

Luis Manuel López Dávila

Universidad San Carlos de Guatemala

Artículo de Reflexión derivado de Investigación.

Recibido: septiembre I de 2015 - Aprobado: octubre 21 de 2015

I Pediatra Neumóloga, Doctora en Educación (Universidad a Distancia de Panamá), Master en Bioética y Derecho (Universidad de Barcelona) Docente y Presidenta del Comité de Bioética (Universidad de Panamá), Presidenta del Comité de Bioética del Hospital del Niño, Panamá; Miembro del Comité Intemacional de Bioética (UNESCO); Docente y Vice-Presidenta REDBIOETICA UNESCO. Dirección postal: 0843-03 I7I, República de Panamá. Dirección electrónica: cverges2004@yahoo.es

2 Licenciada en Ciencias Jurídicas y Sociales (Universidad de Chile), Abogada, docente invitada en Bioética Universidad de Chile, Universidad Andrés Bello. Miembro del Comité de Ética de Investigación del Servicio de Salud Metropolitano Oriente Ministerio de Salud Chile, Miembro del Comité de Ética Clínica y del Comité de Ética de Investigación de Clínica Las Condes, Miembro del Centro de Bioética de la Fundación Cristo Vive y miembro de la Comisión Ministerial de Ética de Investigación en Salud (CMEIS), Ministerio de Salud, Chile. Dirección electrónica: masotomay@gmail.com

3 Licenciada en Ciencias de la Educación (UBA), Especialista en la Problemática del Uso Indebido de Drogas (UBA), Magíster en Salud Pública (UBA), Magíster en Bioética (Universidad de Chile/ OPS) y Doctora en Ciencias Sociales (UBA). DocenteInvestigadora de la Facultad de Medicina de la UBA, Profesora del Diplomado en Bioética (FLACSO) y miembro del Comité de Ética de la Sociedad Argentina de Investigación Clínica (SAIC), de los Comités de Ética del Hospital Tornú y del Comité de Ética del Hospital Elizalde. Dirección electrónica: patriciasorokin@hotmail.com

4 Médico y Cirujano de la Universidad de San Carlos de Guatemala (USAC). Maestría en Población (FLACSO Sede México), Máster en Bioética y Derecho (Universidad de Barcelona), Doctor en Salud Pública (Instituto Nacional de Salud Pública de México). Investigador del Centro de Investigaciones de las Ciencias de la Salud de la (USAC), Coordinador del Comité de Bioética en Investigación en Salud de la USAC; Miembro de la Comisión de Ética y Comité de Bioética en Investigación en Salud de la Facultad de Ciencias Médicas (USAC). Dirección electrónica: luislopez@usac.edu.gt 


\title{
Resumen
}

El desarrollo de biobancos conlleva diversas interrogantes. Nuestro objetivo se circunscribe a cuestiones de confidencialidad y confianza pública en la ciencia y las instituciones. Un problema actual y acuciante en investigaciones biomédicas transnacionales, es la recolección de muestras para almacenamiento y exportación con consentimientos abiertos, con riesgo de violación de la privacidad personal. La necesidad de contar con biobancos para investigación, de proteger el reparto de beneficios de la investigación y la participación de las minorías étnicas presentes en América Latina, nos lleva a proponer una democratización de los biobancos sobre la base de la estructura de Derechos Humanos de la Región, representada por el Pacto de San José de Costa Rica.

Palabras clave: Democratizar, biobanco, privacidad, confidencialidad, datos sensibles, muestras biológicas, investigaciones biomédicas transnacionales, dignidad.

\section{Proposal to "democratize" the benefits of bio-banks in Latin America}

\begin{abstract}
The development of bio-banks carries several questions. Our objective is delimited to confidentiality and public trust issues regarding science and institutions. A current and acute problem in bio-medical international research is the collection of samples for storage and exportation with open consent, with risk of violation of personal privacy. The need to count on research bio-banks, to protect the distribution of research benefits and assuring the participation of Latin American ethnic minorities take us to propose the "democratization" of bio-banks based on the Human rights structure of the region, represented by the Pact of San Jose, Costa Rica.
\end{abstract}

Key words: democratize, bio-bank, privacy, confidentiality, sensitive data, biological samples, transnational biomedical research, dignity.

\section{Propostas para democratizar os benefícios dos biobancos em América Latina}

\section{Resumo}

O desenvolvimento dos biobancos implica diversos interrogantes. Nosso objetivo se circunscreve a questões de confidencialidade e confiança pública na ciência e nas instituições. Um problema atual e urgente nas investigações biomédicas transnacionais é a coleta de mostras para armazenamento e exportação com consentimentos abertos, com riscos de violação de privacidade pessoal. A necessidade de contar com biobancos para investigação, de proteger o reparto de benefícios da pesquisa e a participação das minorias étnicas presentes na América Latina nos leva a propor uma democratização dos biobancos sobre a base da estrutura dos direitos humanos da região, representada pelo Pacto de San José de Costa Rica.

Palavras chave: Democratizar, biobanco, privacidade, confidencialidade, dados sensíveis, amostras biológicas, investigações biomédicas transnacionais, dignidade. 


\section{Introducción}

Las transformaciones científico-tecnológicas del mundo globalizado actual, nos conminan a enfatizar en los problemas éticos que ellas implican. En la práctica se requiere conceptualizar lo que constituye un biobanco, identificar sus usos, el manejo de la información disponible y el acceso, respecto del cual se propone uno amplio, considerando los intereses de las partes involucradas. Entre éstos los beneficios para el sujeto fuente y/o para la comunidad. Se considera el resguardo ético y la protección de las personas sobre la base de una propuesta del acuerdo regulatorio previo de la comunidad internacional que considera el Pacto de San José de Costa Rica, que tiene la ventaja de mantener un sistema permanente de resguardo de sus normas.

Los biobancos representan un nuevo campo de la biotecnología al servicio de la ciencia (investigación genética para la comprensión de la evolución de las especies), de la medicina (tratamiento de las enfermedades monogenéticas) y de la industria farmacéutica (competición comercial para nuevos medicamentos). Investigadores de diferentes países abogan por la creación de biobancos en los países en vía de desarrollo, como alternativa para el control de enfermedades que no han sido controladas con los programas de atención y prevención implementados según las recomendaciones de la Organización Mundial de la Salud (VIH-SIDA, tuberculosis, malaria y enfermedades crónicas de diferentes índoles). La disminución relativa de los costos de mantenimiento del biobanco, gracias a nuevas técnicas de almacenamiento, es otro argumento para ello. ${ }^{5}$ Sin embargo, los países que ya tienen biobancos (China, India, Sud-África) no han resuelto sus grandes problemas de desigualdades en salud. Al respecto, no debe olvidarse que:

La genética ha abierto un mundo de posibilidades individuales y colectivas. La información genética de los sujetos trasciende su individualidad para afectar a la familia y a la sociedad. Pero estas posibilidades no pueden ser analizadas en un contexto hipotético, sino en la perspectiva del sistema económico de libre mercado y en el momento presente, en que la generación de conocimiento está altamente protegida. En este nuevo contexto, las muestras biológicas y la información genética, conjuntamente con el historial clínico, son un elemento de comercio o de acuerdos entre industria y sistema público de salud (como en los casos de Islandia y Estonia) con el objetivo de desarrollar la farmacogenética. Sin embargo, el desarrollo de una posible nueva medicina, tan unido a las corporaciones farmacéutico-sanitarias que utilizan la protección del conocimiento y las patentes para rentabilizar las inversiones, dibuja un horizonte confuso, de difícil análisis y de consecuencias sociales importantes. ${ }^{6}$

5 Sgaier, S. K. et al. Biobanks in Developing Countries: Needs and Feasibility. Sitio Web: Science www.sciencemag.org, Policy Forum, Public Health, 16 November 2007, vol. 318. Búsqueda el 23 de agosto de 20 I5. Disponible en [Internet] http://www.cghr.org/ wordpress/wp-content/uploads/Biobanks-in-developing-countries-needs-and-feasibility-2007.pdf

6 Bota i Arqué, A. "El reto de la muestra biológica en los estudios farmacogenéticos". En Acta bioethica. I0(2), (2004): 20 I-2 I2. Búsqueda el 23 de agosto de 20 I5, Disponible en [Internet] http://www.scielo.cl/scielo.php?pid=SI 726-569X2004000200008\&script=sci arttext 
Dicha circunstancia podría constituirse en un obstáculo para la creación, accesibilidad y/o democratización de los biobancos.

En nuestro continente, ya existen biobancos públicos en Argentina, Brasil, México, Chile y biobancos privados en Panamá, Argentina y Chile, pero hasta el momento la mayoría de los países latinoamericanos y caribeños no cuentan con una regulación específica sobre este tema. Las legislaciones sobre el particular están comprendidas en las leyes sobre bancos de sangre o trasplante. Países como Colombia y México $^{8}$ han solicitado la reflexión de las instituciones nacionales de bioética para una regulación jurídica.

El mayor problema que se enfrenta en la actualidad, reside en la recolección de muestras para exportación a través de consentimientos abiertos anexados a las investigaciones biomédicas transnacionales, lo cual constituye un abuso de la confianza que se deposita en el investigador por parte del paciente y potencialmente puede llegar a una violación de la privacidad de los pacientes. Frente a esta situación, la Bioética nos confronta a proponer alternativas para el desarrollo de regulaciones protectoras, específicamente los derechos de las personas de nuestro continente. ${ }^{9}$

El Convenio de Biomedicina y Derechos Humanos en su artículo $10^{\circ}$ y la Declaración Universal sobre Bioética y Derechos Humanos en su artículo $9^{\circ}$, entre otros, receptan el respeto y el resguardo de la privacidad y de la confidencialidad.

\section{Bioética y Derecho}

Las sociedades necesitan normas para funcionar y prevenir, regular y sancionar los problemas que derivan del desarrollo de cualquier institución, comercio o industria que afecta a la ciudadanía en su vida cotidiana y de las futuras generaciones. El debate bioético debe preceder a la adopción de estas leyes como resultado de un consenso social. La biotecnología, por su novedad y complejidad, implica un desafío importante en lo jurídico.

Con anterioridad al trabajo de UNESCO en sus Declaraciones de 1997, 2003 y 2005, la comunidad internacional representada en Naciones Unidas, ya había reconocido normas vinculantes en relación con el avance de la biotecnología. Así el Convenio sobre la Diversidad Biológica de 1992, que tiene como objetivo la promoción de medidas conducentes a un futuro sostenible, se propone la conservación de la diversidad biológica, la utilización sostenible de sus componentes y la participación

7 Veloza C., Luis Alberto; Wiesner C., Carolina; Serrano L., Martha Lucía; Peñaranda C., Nelsy Ruth; Huertas S. Antonio. Consideraciones éticas y legales de los biobancos para investigación. Revista Colombiana de Bioética, vol. 5, núm. I, enero-junio, 20 I0, pp. I2 I- I 4I, Universidad El Bosque Bogotá, Colombia, Búsqueda el 23 de agosto de 20 I5, Disponible en [Internet] http:// www.redalyc.org/articulo.oa?id= 189217244008

8 Soto G., Liliana. Regulating Mexican biobanks for human biomedical research: what can be learned from the European experience? MEXICAN LAW REVIEW Vol. VII, No. I, 3I-55, 20 I4. Búsqueda el 23 de agosto de 20 I 5, Disponible en [Internet] http://biblio. juridicas.unam.mx/revista/pdf/MexicanLawReview/I3/arc/arc2.pdf

9 Justo, Luis. Extractivismo genético en América Latina y el Caribe, Revista Redbioética/UNESCO, Año 4, I (7): X-XX, Enero Junio 2013, 9-10. ISSN 2077-9445 
justa y equitativa en los beneficios que se deriven de la utilización de los recursos genéticos. Además de la diversidad genética, son componentes de la diversidad biológica todas las formas de vida que hay en la Tierra, incluidos ecosistemas, animales, plantas, hongos y microorganismos. ${ }^{10}$

Se considera que jurídicamente el material biológico no es una mera "cosa". Al respecto, la Declaración Internacional sobre los Datos Genéticos Humanos establece que: "habría que aplicar las mismas rigurosas exigencias de confidencialidad a todos los datos médicos, comprendidos los datos genéticos y los proteómicos, con independencia de la información que aparentemente contengan...(y) se debería prestar la debida atención al carácter sensible de los datos genéticos humanos e instituir un nivel de protección adecuado de esos datos y de las muestras biológicas." "I

El manejo inadecuado, tanto del material biológico como de la información genética puede resultar dañoso para el sujeto fuente, para su familia o eventualmente para la comunidad.

La colección organizada de muestras humanas se conoce en general como biobanco. A efectos de este trabajo, consideraremos la definición dada por Veloza Cabrera, como "una colección organizada de muestras biológicas y los datos asociados a ellas, almacenadas con fines diagnósticos, terapéuticos o de investigación, indistintamente del grado de identificación de las muestras y datos"'|2 a la cual agregamos que puede ser un establecimiento público o privado, con o sin ánimo de lucro. Tanto la colección, como el almacenamiento y el tipo de establecimiento afectan a la privacidad, y confidencialidad de los individuos y grupos sociales o étnicos.

La Bioética implica la expresión de la pluralidad y diversidad de las opciones morales presentes en las sociedades actuales, para el logro de un consenso de mínimos que serán representados en las leyes de un país. ${ }^{13}$ Desde el enfoque universal de Derechos Humanos, la Bioética permite la reflexión de temas que transcienden en contextos donde la ausencia de marcos de regulación son la base de vulneración a los derechos ciudadanos. Es en este contexto de ausencia de leyes, que el abuso de confianza y la violación a la privacidad pueden suceder, bajo el subterfugio de la aplicación de un consentimiento informado con fines inciertos o no explicitados que trasciende las fronteras para el traslado de muestras, producto de la experimentación en humanos.

Por su parte, los Derechos Humanos, asentados en el reconocimiento de la dignidad inherente a todos los miembros de la familia humana y de sus derechos iguales e inalienables, consideran en el Pacto Internacional de Derechos Económicos Sociales y Culturales ${ }^{14}$ el derecho de toda persona

10 Naciones Unidas, Convenio sobre la Diversidad Biológica, 1992. Búsqueda el 30 de agosto de 20I5, Disponible en [Internet] https://www.cbd.int/doc/legal/cbd-es.pdf

I UNESCO, 2003. Los destacados en bastardilla nos pertenecen.

12 Veloza C., Luis Alberto; Wiesner C., Carolina; Serrano L., Martha Lucía; Peñaranda C., Nelsy Ruth; Huertas S. Antonio. Op. Cit.

13 Casado G., María. ¿Por Qué Bioética y Derecho?, Rev. Acta Bioethica 2002; año VIII, n², I43-193, pág. 191

14 Naciones Unidas, Pacto Internacional de Derechos Económicos, Sociales y Culturales, 1966, Búsqueda el 23 de agosto de 20 I5, Disponible en [Internet] http://www.un.org/es/events/culturaldiversityday/covenant.shtml 
al goce de los beneficios del progreso científico y de sus aplicaciones y a beneficiarse del fomento y desarrollo de la cooperación y de las relaciones internacionales en cuestiones científicas y culturales.

Los Derechos Humanos abordan principalmente la relación entre los individuos y el Estado. Las obligaciones gubernamentales con respecto a los Derechos Humanos en términos generales se engloban en los principios de respeto, protección y realización personal, como lo ha reconocido respecto de la salud la OMS. ${ }^{15}$ Además, asentados en el reconocimiento de la dignidad inherente a todos los miembros de la familia humana y de sus derechos iguales e inalienables, el Pacto Internacional de Derechos Económicos Sociales y Culturales ${ }^{16}$ releva el derecho de toda persona al goce de los beneficios del progreso científico y de sus aplicaciones y a beneficiarse del fomento y desarrollo de la cooperación y de las relaciones internacionales en cuestiones científicas y culturales. La posibilidad de facilitar el acceso de las personas a biobancos, se inserta en los principios señalados.

Las Constituciones Políticas de los países de América Latina de opción democrática, se apoyan en el respeto a los Derechos Humanos, principalmente: la libertad (traducida en Bioética como la autonomía de decisión de las personas y el deber de respeto de estas decisiones por parte de los demás) y la integridad (dignidad y respeto de la unidad de la persona en un concepto holístico), el derecho a la salud (como deber del Estado de facilitar las necesidades básicas para el desarrollo humano y especialmente el acceso a servicios de salud de calidad, así como el deber de protección de las y los ciudadanos).

En relación con el aprovechamiento compartido de los beneficios (benefit sharing), el artículo I 5 de la Declaración Universal sobre Bioética y Derechos Humanos sostiene que:

"Los beneficios resultantes de toda investigación científica y sus aplicaciones deberían compartirse con la sociedad en su conjunto y en el seno de la comunidad internacional, en particular con los países en desarrollo. Los beneficios que se deriven de la aplicación de este principio podrán revestir las siguientes formas:
a) asistencia especial y duradera a las personas y los grupos que hayan tomado parte en la actividad de investigación y reconocimiento de los mismos;
b) acceso a una atención médica de calidad;
c) suministro de nuevas modalidades o productos de diagnóstico y terapia obteni- dos gracias a la investigación;
d) apoyo a los servicios de salud;
e) acceso a los conocimientos científicos y tecnológicos
f) instalaciones y servicios destinados a crear capacidades en materia de investigación;

\footnotetext{
I5 OPS/OMS, Recomendaciones sobre la ética de la prevención, atención y control de la tuberculosis. Programa Regional de Bioética, Oficina de Género, Diversidad y Derechos Humanos. Washington, 20I3. Búsqueda el 30 de agosto de 20I5, Disponible en [Internet] http://apps.who.int/iris/bitstream/I 0665/89637/I/9789275317433_spa.pdf

16 Naciones Unidas, 1966. Op. Cit.
} 
g) otras formas de beneficio compatibles con los principios enunciados en la presente Declaración.

2. Los beneficios no deberían constituir incentivos indebidos para participar en actividades de investigación."

Asimismo, los intentos de acuerdos para compartir beneficios ${ }^{17}$ se han ido convirtiendo -paulatinamente- en uno de los temas de mayor convocatoria y controversia de los foros internacionales.

Con esta visión, el reconocimiento constitucional hace parte del marco jurídico referencial frente a cualquier violación de los derechos de las personas que entregan sus muestras para investigación inmediata o conservación para investigaciones futuras (es necesario entender y difundir que los biobancos para "conservación" representan un depósito de muestras biológicas para investigaciones futuras ya que tienen pocas aplicaciones médicas actuales). En particular, el derecho a la integridad y la dignidad, conlleva el derecho a la privacidad de las personas interesadas y a la confidencialidad de la información que les atañe. Para disponer de la información personal o datos personales y de la totalidad o partes del cuerpo, la persona debe dar su consentimiento luego de conocer y comprender los objetivos de la donación. Pero este consentimiento no es suficiente dado que la sensibilidad personal y social de los datos íntimos y de las muestras de una persona, obliga a los recolectores y conservadores de estas, al máximo cuidado en cuanto a la confidencialidad y reserva de la información, que forma parte de lo que se denomina "datos sensibles" habida cuenta de su potencial discriminador.

Por otra parte, la información así obtenida no debería utilizarse para fines distintos de los que determinaron su acopio o para los que se obtuvo el consentimiento, de conformidad con el Derecho Internacional, en particular el relativo a los derechos humanos. Así lo subraya la Declaración Universal sobre Bioética y Derechos Humanos (DUBDH) $)^{18}$ en su artículo $9^{\circ}$ y las Declaraciones sobre el Genoma Humano y los Derechos Humanos ${ }^{19}$, 20. La DUBDH desglosa los derechos específicos de las y los participantes en las investigaciones, así como los deberes del Estado y de la sociedad para el respeto de la privacidad y confidencialidad de las personas involucradas. Aunque las dos últimas Declaraciones no se refieren nominalmente a los biobancos, señalan las obligaciones éticas de la recolección y el almacenamiento de material genético.

Las Declaraciones Internacionales señaladas, se inscriben en los propósitos de UNESCO, en el sentido de promover el respeto de la dignidad humana y proteger los derechos humanos, de reconocer

17 Hugo Ethics Committee, Statement on Benefit -Sharing, Vancouver 9 de Abril de 2000. Búsqueda el 3I de agosto de 20।5, Disponible en [Internet] http://www.hugo-international.org/img/benefit_sharing_2000.pdf

I8 UNESCO, DUBDH, 19 de octubre de 2005, Búsqueda el 23 de agosto de 20 I5, Disponible en [Internet] http://portal.unesco. org/es/ev.php-URL_ID=3 I 058\&URL_DO=DO_TOPIC\&URL_SECTION=20 I.html

19 UNESCO, DUGHDH, II de noviembre de 1997, Búsqueda el 23 de agosto de 20I5, Disponible en [Internet] http://portal. unesco.org/es/ev.php-URL_ID=| 3177 \&URL_DO=DO_TOPIC\&URL_SECTION=20 I.html

20 Ver sobre los Datos Genéticos Humanos en: UNESCO, DUDGH, I6 de octubre de 2003, Búsqueda el 23 de agosto de 2015 , Disponible en [Internet] http://portal.unesco.org/es/ev.php-URL_ID=|7720\&URL_DO=DO_TOPIC\&URL_SECTION=20 I. html 
la importancia de la libertad de investigación científica y las repercusiones beneficiosas del desarrollo científico y tecnológico; y de promover el acceso equitativo a los adelantos de la medicina, la ciencia y la tecnología en el marco de principios éticos, entre otros propósitos. Si bien estas declaraciones no tienen carácter obligatorio como los Pactos citados anteriormente, representan compromisos de los Estados miembros de Naciones Unidas, en el sentido de promover tales principios por medios diversos. Algunas constituciones, como la de Panamá21, reconocen su impacto jurídico cuando han sido suscritas por el país. Otras como la de Chile requieren la evolución de las Declaraciones hacia Tratados, Pactos o Convenciones, las que al recaer en derechos humanos, constituyen limitaciones a la soberanía del Estado. ${ }^{22}$

Conforme sostienen tanto el Consejo de Organizaciones Internacionales de Ciencias Médicas (CIOMS) como la UNESCO, en caso que se desee realizar otros estudios respecto de muestras obtenidas con antelación debe obtenerse un nuevo consentimiento. Pero, nótese que no sería necesario volver a solicitar el consentimiento si se tratara del uso de muestras biológicas conservadas, siempre que sea protegida la privacidad de los datos y se preserve el anonimato de la persona a la cual pertenece esa información. Se requiere además la aprobación de un Comité de Ética conforme a lo previsto en la Declaración Internacional sobre Datos Genéticos Humanos (DIDGH).

La Organización Mundial de la Salud ${ }^{23}$ considera adecuado el empleo del consentimiento general para futuros usos de las muestras en investigación, exclusivamente cuando los datos no puedan ser identificables y, excepcionalmente, si se tratara de muestras y de datos codificados. ${ }^{24}$ Pero, si las muestras fueran doblemente disociadas, sería imposible recontactar a los participantes y éstos no podrían beneficiarse de los resultados de las investigaciones ni se les podría realizar un seguimiento que permita monitorear y evaluar su estado de salud como así tampoco agregar información a las bases de datos. Como puede apreciarse, el tema es complejo y controversial, especialmente en dos de los aspectos que integran la definición de biobanco: la ausencia de un fin de lucro y el destino de la información colectada.

Para la circulación internacional de datos y de muestras biológicas en el marco de atención médica, la regla prevalente es la del país donde se obtuvo la muestra, y los acuerdos multilaterales sobre investigación deberían respetar esta regla. ${ }^{25}$ Es evidente que las y los ciudadanos de los Esta-

21 Constitución Política de la República de Panamá Reformada por los Actos Reformatorios de 1978, el Acto Constitucional de 1983 y los Actos Legislativos de 1994 Art. 4, Epasa, I5 noviembre 2004, Gaceta Oficial. Búsqueda el 23 de agosto de 2015, Disponible en [Internet] http://www.epasa.com/constitucion/constitucion.pdf

22 Constitución Política de la República de Chile Artículo 5. Búsqueda el 23 de agosto de 20।5, Disponible en [Internet] http:// www.leychile.cl/Navegar?idNorma $=242302$

$23 \mathrm{WHO}$, Genetic databases: Assessing the benefits and the impact on human rights and patients' rights. s/f, Búsqueda el 23 de agosto de 2015, Disponible en [Internet] http://www.codex.vr.se/texts/whofinalreport.rtf

24 Knoppers, B. Biobanking: International Norms. Journal of Law, Medicine \& Ethics, Vol. 33 (I). pp. 7- I4. John Wiley \& Sons, Inc., 2005.

25 Soto G., Liliana. Regulating Mexican biobanks for human biomedical research: what can be learned from the European experience? MEXICAN LAW REVIEW Vol. VII, No. I, 3I -55, 20 I4. Búsqueda el 23 de agosto de 20 I 5, Disponible en [Internet] http://biblio. juridicas.unam.mx/revista/pdf/MexicanLawReview/ / 3/arc/arc2.pdf 
dos en los cuales rigen políticas de salud y derechos humanos, estarán más protegidos que los que viven en Estados sin este marco legal o donde prevalece una visión de mercado. No obstante, la ya mencionada Declaración Internacional sobre los Datos Genéticos Humanos alerta sobre la "creciente importancia de los datos genéticos humanos en los terrenos económico y comercial (enfatizando que) la recolección, el tratamiento, la utilización y la conservación de los datos genéticos humanos pueden entrañar riesgos para el ejercicio y la observancia de los derechos humanos y las libertades fundamentales y para el respeto de la dignidad humana".

Bajo el principio de solidaridad se debería de plantear un biobanco de acceso universal al considerar las diferencias económicas que el modelo de desarrollo capitalista establece entre los países de la región. Según Kottow y Schramm ${ }^{26}$ la solidaridad "asume que la atomización de un colectivo hace más vulnerable a cada uno de sus miembros, por lo cual es recomendable entender este colectivo como un sólido (= solidaridad) que aúna los esfuerzos de todos para paliar infortunios, defenderse de agresiones, constituir un sistema de protección y elaborar en conjunto para la obtención de bienes comunes."

Dos aspectos son relevantes en materia de democratización con respecto a la economía periférica: por un lado, la brechas de acceso al conocimiento y la tecnología, y por el otro el marco legal donde se sustentan los derechos individuales y colectivos. Éste se podría sostener en un acuerdo continental, sobre la base del Pacto de San José de Costa Rica, el cual podría ofrecer amparo para todos los habitantes de América Latina, considerando las etnias, su cultura y biodiversidad, con la protección de la Corte Interamericana de Derechos Humanos.

Asimismo, la Declaración Universal sobre Bioética y Derechos Humanos insiste sobre la solidaridad entre naciones (transferencia de tecnología y conocimientos) y sobre la responsabilidad social de los Estados y de los ciudadanos en cuanto al acceso a los servicios de salud (Art. 13 y |4) (Art. I 3 y |4) para todas las personas teniendo en cuenta "la igualdad fundamental de todos los seres humanos" (Art. 10).

Hay otros temas relacionados, en este caso con la información genética que conminan al Derecho, así "su depósito y la circulación a través de muestras biológicas guardadas en biobancos, las historias clínicas informatizadas que contienen datos genéticos personalizados, la utilización de perfiles genéticos humanos en medicina forense y la creación de ficheros destinados a su guarda y conservación. Ubicados en esta realidad, advertimos que la información genética humana merece un tratamiento singular por parte del Derecho, en atención a los múltiples efectos que puede tener para el individuo, su circulación y tratamiento fuera de su esfera de control'27.

26 Schramm Fermin Roland, Kottow Miguel. Principios bioéticos en salud pública: limitaciones y propuestas. Cad. Saúde Pública [Internet]. 200I Aug 17(4): 949-956. Búsqueda el 4 de Diciembre de 2015 Disponible en [Internet] http://www.scielo. br/scielo.php?pid=S0102-31 IX200 1000400029 \&script=sci arttext

27 Bergel, S. D.: Información, dato y perfil genéticos ante el derecho. En Revista Crítica de Derecho Privado, Número II (20 I4), págs. | 35 |- 1384. 


\section{Análisis de la situación y propuestas iniciales}

\section{Privacidad,confidencialidad y confianza}

El Diccionario de la Real Academia Española considera que la privacidad es el "ámbito de la vida privada que se tiene derecho a proteger de cualquier intromisión"'28. Este derecho se desprende del reconocimiento de la dignidad y de la libertad individual de todo ser humano. Sobre esta base, es esencial que toda decisión con respecto a los biobancos sea ampliamente consultada con todas las personas que puedan ser llamadas a participar en investigaciones con muestras y datos genéticos o que estarán confrontadas a publicidades sobre conservación de las mismas para tratamientos futuros. La preservación de la privacidad hace parte de las obligaciones éticas como modalidad de convivencia en respeto mutuo y paz. Además conlleva consigo la confianza de las personas (usuarias o no) de los servicios de salud vigilados por el Estado como garante de sus derechos.

Así entonces, que el primer paso sería obtener y mantener la confianza de la población en el objeto de nuestra reflexión que son los biobancos, sean éstos para investigación o para transplante. La confianza se gana cuando los investigadores, los patrocinadores y las autoridades gubernamentales involucradas muestran la máxima responsabilidad, prudencia y transparencia en sus decisiones. ${ }^{29}$ En algunos países de Latinoamérica y el Caribe, la confianza ha sido lesionada por decisiones autoritarias o paternalistas. Los escándalos de investigaciones nacionales e internacionales reportados en los últimos años (investigación sin información ni consentimiento de poblaciones vulnerables en Guatemala, investigación de la vacuna contra el neumococo sin consentimientos adecuados en Argentina, entre otros casos) $)^{30,31}$ han extendido esta desconfianza al campo de la ciencia y a todo lo que se asocia con ella. Por otra parte la oposición de ciertos grupos de poder y de presión a favor o en contra de la genética y el uso de células madres de embriones, puede aumentar la confusión general.

Se debe igualmente tomar en cuenta que "los usos secundarios de las muestras humanas almacenadas son casi siempre posibles aunque usualmente no son predecibles al momento de toma de la muestra. Las problemáticas éticas preponderantes se relacionan con el nivel de completitud de la información dada, la necesidad o no de obtener un nuevo consentimiento individual para cada uso, y con quien decide en este asunto. Diversos puntos de vista han sido expresados, desde el denegar todo uso que no sea el originalmente dado, a actitudes más flexibles. Esto último toma en consideración la trazabilidad (rastreabilidad) o no de la identidad individual, el tipo de usos futuros en relación

28 Diccionario de la lengua española 22. edición y las enmiendas incorporadas hasta 20I2. Búsqueda el 23 de agosto de 2015 , Disponible en [Internet] http://lema.rae.es/drae/

29 Vergès, Claude. Genética y Bioética en América Latina, Rev. Acta Bioethica 2004; año X, No. 2, págs. I55-I66.

30 López Dávila, Luis; Rodríguez, Virginia; Sagastume, Marco Antonio; Peralta, Andres; Siedel, Liliana; Guilghem, Dirce. El caso "Guatemala". En: Sorokin, Patricia. (coord.). Número especial de jurisprudencia argentina sobre derechos personalísimos y VIH/ sida en el nuevo decenio. AbeledoPerrot, Buenos Aires, Abeledo Perrot; 20 I I, págs. 8I-8.

31 Tealdi, J. C. Problemas bioéticos en la investigación de nuevas vacunas: ¡obedecen a razones de salud pública? Salud colect. Lanús, v. II, n. I, marzo 2015. Búsqueda el 26 de agosto de 2015, Disponible en [Internet] http://www.scielo.org.ar/scielo. php? script=sci arttext\&pid=S| $85 \mid-82652015000100007 \& \operatorname{lng}=$ es\&nrm=iso 
con el uso original, las implicaciones de la investigación del individuo (llamado investigación de `riesgo mínimo'), cuan preciso fue descrito el uso al momento de tomar la muestra, y finalmente, el tipo de consentimiento concedido originalmente". ${ }^{32}$

Tomando modelo sobre la investigación participativa con miembros de comités de ética de investigación en Botswana ${ }^{33}$, las preguntas esenciales serían: “QQué piensan los diferentes grupos ciudadanos de la recolección de muestras biológicas y datos personales? ¿Qué piensan de su conservación en general o después de terminar una investigación?"

En el ámbito latino-americano los biobancos pueden ser nacionales (países con gran desarrollo de la investigación y con capacidad nacional instalada) o por asociaciones de países (pocos habitantes, pobreza, postguerra). Aunque los biobancos representen una necesidad de la investigación frente al desarrollo internacional de la genética y de la biotecnología, pueden no representar una prioridad nacional frente a problemas de salud no resueltos, con lo cual la cooperación entre países del continente puede ser un ejemplo de la solidaridad entre pares. Por otra parte, la percepción de la privacidad de los datos personales, así como de la imagen de la integridad del cuerpo y su relación con sus componentes biológicos, ha sido poco estudiada en la mayoría de los países y merece ser considerada tanto para el problema específico de los biobancos como para la atención médica en los servicios de salud.

Algunos países han desarrollado prácticas de diálogo social para el diseño de políticas relativas a la vida de sus ciudadanos: fertilización asistida en Argentina por ejemplo, o eutanasia en Colombia. Otros desconocen esta práctica y los medios de comunicación se orientan a temas más llamativos, que a la discusión sobre temas de difícil manejo y probablemente poca comprensión. El nivel de conocimiento en ciencia y la difusión de publicidades engañosas sobre remedios "milagrosos" complican el debate. Sin embargo, los biobancos privados se instalan de hecho en muchos países, aprovechando la ausencia de leyes específicas que los limiten o regulen, como también aprovechando la libertad que brinda el Derecho Privado en que lo que no está prohibido está permitido. Por su parte, el sector público está escaso de recursos y no vislumbra esta necesidad como inminente. En el contexto señalado, esta convocatoria constituye entonces una necesidad independiente de otras prioridades sanitarias.

\section{¿Biobancos públicos o privados?}

La consideración o pregunta sobre el carácter público o privado de los biobancos tiene importancia en relación con el acceso a la privacidad de los datos y muestras. La ventaja de los biobancos públicos

32 Cambon-Thomsen, Anne. The social and ethical issues of post-genomic human biobanks. Nature (5), 2004: 867-873. Búsqueda el 26 de agosto de 20 I 5, Disponible en [Internet] https:/eclass.duth.gr/modules/document/file.php/ALEX01 196/The\%20social\%20 and\%20ethical\%20issues\%20of\%20post-genomic\%20human\%20biobanks.pdf

33 Barchi, Francis et al. "The keeping is the problem": A qualitative study of IRB-member perspectives in Botswana on the collection, use, and storage of human biological samples for research. BMC, Medical Ethics, 19 de agosto de 20I5, I6:54 DOI 10.1 I86/s I29I00 15-0047-3. Búsqueda el 23 de agosto de 20 I5, Disponible en [Internet] http://www.biomedcentral.com//472-6939/16/54 
es que el Estado tiene una obligación reconocida de proteger a todos sus ciudadanos en igualdad y de rendir cuentas por sus acciones. Aunque la igualdad ciudadana está plasmada en las constituciones nacionales de los países que hemos tomado como ejemplo, las desigualdades socio-económicas están presentes en mayor o menor grado en todos ellos. La falta o dificultades de acceso a los servicios de salud en general y particularmente a los biobancos, representa una faceta de esta desigualdad, a la cual debe responder el Estado a través de biobancos públicos.

La vigilancia ciudadana respecto al acceso de las personas individuales a sus propios datos y de la conservación apropiada de los mismos, es extrema en cuanto se trata de la "cosa pública" y la responsabilidad política que conlleva; y debido también a la cada vez mayor conciencia del ciudadano respecto de sus derechos. Esto supone mecanismos de rendición de cuentas que existen actualmente en leyes de transparencia de muchos países; y la vigencia y operatividad independiente de los Comités de Bioética. En particular, los comités deben vigilar que no se utilicen las intervenciones en nombre de prioridades de salud pública sin las debidas justificaciones y que los biobancos generales sean totalmente separados de los biobancos de medicina forense. Los intercambios de informaciones entre cada uno pueden darse en ocasiones, pero deben tener una sustentación irrefutable. La permanencia de los biobancos públicos supone también, un presupuesto de salud o de investigación, que está lejos de la realidad actual de muchos países.

La ventaja proclamada de los biobancos privados, reside en el traslado de los costos de mantenimiento a la entidad propietaria de la instalación. Las políticas de privacidad de las muestras y de la confidencialidad de los datos hace parte del buen nombre de esta entidad y generalmente se cumple, al igual que en los bancos financieros. El problema es justamente la figura de un banco financiero privado: de compra y venta de los datos y/o del material biológico de la misma manera que se hacen las transacciones monetarias, inclusive en las bolsas internacionales (recordemos la evolución de Facebook, Google y otros bancos de datos en Internet). Frente a este peligro, las leyes nacionales y el marco jurídico internacional deben ser lo más estrictos y claros posibles para la protección de las personas, de sus datos y muestras biológicas, así como sobre la distribución de los riesgos y beneficios. Teniendo presente la Declaración Universal sobre el Genoma Humano y la Declaración sobre Datos Genéticos de UNESCO, consideramos que se debe prohibir la comercialización de las muestras biológicas y datos personales en todos sus aspectos para mantener la privacidad de cada persona. Esta privacidad absoluta no impide el altruismo y la solidaridad al compartir estos datos con investigadores e instituciones, siendo oportuno y necesario poner de relieve aquí que la Declaración Universal sobre Bioética y Derechos Humanos establece que "los Estados deberían tomar las medidas adecuadas en los planos nacional e internacional para luchar contra el bioterrorismo, así como contra el tráfico ilícito de órganos, tejidos, muestras, recursos genéticos y materiales relacionados con la genética."

Ambos sistemas, sea el público o el privado suponen también, una capacidad de supervisión que necesita un compromiso jurídico y ético así como los conocimientos científicos en genética y biotecnología, para el manejo de estas muestras y datos por parte de los funcionarios o miembros de los Comités de Bioética reconocidos por las autoridades y la ciudadanía; la capacidad de emitir 
pronunciamientos que puedan implicar la aplicación de sanciones; y un presupuesto que asegure un trabajo eficaz.

\section{Voluntariedad y consentimiento informado de los donantes}

La regulación aplicable debe asegurar también la voluntariedad y el consentimiento de los donantes (con el detalle de la información recibida), con acceso permanente a sus datos/muestras y posibilidad de retiro y destrucción, con objetivos definidos (conservación e investigación).

- Una vez definido el tipo de biobancos que permitirá el país, se inicia una fase pre-operativa de mucha importancia y que debe tomar el tiempo necesario para formular todas las consultas éticas, médicas y jurídicas nacionales e internacionales. Es importante la experiencia de los países industrializados, pero también la de los países con problemas económicos, ecológicos y con políticas similares. Para considerar esta etapa, nos apoyaremos en la Declaración Internacional sobre los Datos Genéticos Humanos ${ }^{34}$

\section{Propuestas generales para la "Democratización” desde los Derechos Humanos}

La democracia implica construir la sociedad con la participación de todos sin discriminación. Los Derechos Humanos sirven de marco referencial legal para este propósito y la bioética aporta sus valores éticos y su metodología de dialogo para la democratización de cualquier servicio de salud, en este caso de los biobancos. Dicho lo anterior, se requiere entonces considerar:

\section{I.- La creación de Comités de Ética Independientes, interdisciplinarios y pluralistas en} los planos nacional, regional, local o institucional (Art. 6, b). Los Comités de Ética son organismos colegiados cuya función es vigilar el buen cumplimiento de las leyes y reglamentos para la protección de los derechos de los y las participantes en investigaciones (Comités de Ética en investigación) y de los y las pacientes en la atención clínica (Comités de Ética asistenciales). Ambos deben también reflexionar sobre los nuevos problemas de la ciencia y de la medicina y su impacto sobre los seres humanos, los animales y el medio ambiente. Uno de los derechos principales es el respeto a la privacidad individual, de la familia o de un grupo particular en las relaciones arriba mencionadas y su corolario es el respeto de la confidencialidad de los datos privados entregados ${ }^{35}$ En este sentido la Declaración de la Asociación Médica Mundial sobre las Consideraciones Éticas de las Bases de Datos de Salud de 2002, estipula en su art. 14 que "se debe obtener la aprobación de un comité de revisión ética especialmente nombrado para toda investigación en la que se utilice información de pacientes,

34 UNESCO, 2003. Op. Cit.

35 AMM, Declaración de la AMM sobre las Consideraciones Éticas de las Bases de Datos de Salud, 53a Asamblea General, Washington, 2002. Búsqueda el 26 de agosto de 20 I5, Disponible en [Internet] http://www.wma.net/es/30publications/I0policies/d l/ 
incluida la investigación nueva que no estaba prevista al momento de recopilar la información." Los Comités de Ética son los garantes de la privacidad y confidencialidad de los datos y muestras recogidas y su independencia refuerza la confianza de los y las donantes, y la sociedad en general en que estos datos no serán violados.

La independencia se establece en las leyes y reglamentos que reconocen la libertad de decisiones de estos comités y su autonomía financiera. La permanencia de esta independencia dependerá de la capacidad del comité de justificar sus decisiones de manera transparente y responsable, mostrando su capacidad de resistir a las presiones financieras, políticas y académicas. Si bien la ley puede establecer los criterios de esta transparencia y responsabilidad, solo los miembros del comité son capaces de responder a estos criterios. El ejemplo de países con Comités "independientes" formados por profesionales al servicio de los patrocinadores o incapaces de resistir a las presiones, ha retrasado la investigación en los mismos. ${ }^{36}$ La reproducción de estas fallas en el manejo de los biobancos, sería una violación del respeto a los donantes y a la ciudadanía en general, implicando un retraso para los beneficios que aporta la genética a la medicina. Aunque concordamos con este enunciado, llamamos la atención para que no se quede solo como una aspiración o deseo ideal.

2. Revocación de consentimiento y acceso (Arts. 9 y 13). La revocación del consentimiento es un derecho inherente a la libertad de decisión de cada persona y está considerada en la DUBDH, la Declaración de Helsinki y las Guías de la CIOMS para la investigación con seres humanos. Su aplicación en el caso de muestras identificables, está ampliamente definida en la Declaración Universal. La confianza de los y las participantes en los biobancos se consolida con el respeto de este derecho, la simplicidad de los trámites para concretarlo y la ausencia de represalias por esta decisión. Los problemas prácticos de la revocación de consentimiento están ligados a las medidas tomadas para preservar la privacidad y la confidencialidad de los datos y las muestras biológicas.

3. Privacidad y confidencialidad: El problema se inicia cuando las muestras ya no son identificables (investigaciones o muestras de muchos años). La Declaración así como las guías sobre ética de la investigación (AMM 2002, art. 17), insisten en la disociación de las muestras y la identificación de los participantes, a fin de preservar su privacidad y confidencialidad. Esto se traduce en la aplicación de código para los datos y las muestras, dirigidos a la búsqueda de la máxima objetividad en el manejo de los resultados. Para ello, en general las investigaciones clínicas mantienen dos registros independientes manejados por investigadores diferentes: un registro de los datos de identificación personal y el código correspondiente; y un registro de los datos necesarios para la investigación con su código. Estos registros deben conservarse durante un tiempo específico, a fin de poder revisar cualquier duda o para supervisión de las autoridades (Comité de Ética de Investigación, autoridades gubernamentales). Concluida la investigación, los registros deben destruirse. Podríamos imaginar un sistema de registro similar para los biobancos fijando un tiempo determinado por criterios técnicos (calidad de conservación y posibilidad de uso) y éticos (periodicidad del consentimiento informado del/la donante.

36 Duro, Eduardo y Torres, Susana. ¿Qué se entiende, aquí y ahora, por “Comités de Ética independientes"? En F. León Correa y P. Sorokin (comps.) Bioética y Salud Pública en y para América Latina. FELAIBE: Santiago de Chile, 20 I5: págs. 459-463. 
4. Biobancos como parte del sistema público de salud. El ejemplo del Reino Unido y de España es importante dado que los biobancos nacionales son parte del sistema público de salud y están alojados en hospitales universitarios dedicados a la investigación genética y clínica sobre los problemas prioritarios del país ${ }^{37}$. Esta posición permite el acceso a todas las personas que desean hacer uso de sus muestras, sin discriminación de ninguna índole. La privacidad de los datos y muestras está preservada por protocolos estrictos conocidos por los donantes y por los investigadores, con un sistema de codificación a cargo de equipos diferentes bajo la vigilancia de un Comité de Ética independiente, asesor de la dirección del biobanco. Siendo un referente para la investigación nacional e internacional, todos los protocolos de investigación solicitando el acceso a estos biobancos son examinados cuidadosamente por un comité científico y un comité ético. Desde el inicio, los donantes saben que sus muestras biológicas serán utilizadas para investigaciones, que la administración y el comité de ética son garantes de la confidencialidad y privacidad de sus datos, que pueden tener acceso a ellos en todo momento, así como retirar su consentimiento y estar seguros de que sus muestras serán destruidas en este biobanco. ${ }^{38}$ El éxito del biobanco español y la confianza lograda en el país ha permitido extender la recolección a los menores de 18 años, introduciendo además del consentimiento de los padres o tutores legales, el asentimiento dinámico y continuo de estos menores conforme a su evolución psicológica, y los mismos derechos de privacidad, confidencialidad y retiro que los adultos. ${ }^{39}$ La confianza en el uso de las muestras para investigaciones está dada también por el conocimiento de los donantes de que sus muestras biológicas son accesibles a todos los investigadores a un costo justo correspondiente al mantenimiento y transporte y de que su donación altruista da una oportunidad a las personas enfermas (principio de solidaridad). Sin embargo, esta posición está cuestionada por académicos de Estados Unidos, que se apoyan en la anonimización de las muestras y en la historia de los bancos de sangre. Así, autores como Klara Swanson ${ }^{40}$ proponen un pago a los donantes, haciendo el parangón con el equilibrio entre entradas y salidas de estos bancos y de los bancos financieros. Conociendo el problema mundial de tráfico de órganos que afecta principalmente a los países pobres y con poca capacidad de vigilancia y protección de sus ciudadanos, la propuesta de compra-venta resulta anti-ética porque sería unidireccional de pobres a ricos. En nuestra propuesta de democratización de los biobancos, proponemos que los beneficios para los donantes sean el acceso a la información sobre la comprensión y el tratamiento de las enfermedades investigadas, y el acceso a los medicamentos derivados de su donación cuando los necesiten. Para la sociedad de un país en el cual se ha desarrollado la investigación a partir de los datos y muestras de

37 Williams, Rachel; Mant, Christine; Cason, John. Commentary The Infectious Diseases BioBank at King's College London: archiving samples from patients infected with HIV to facilitate translational research. Open Access Retrovirology 2009, 6:98 doi: I 0. I I 86/I 7424690-6-98 Búsqueda el 26 de agosto de 20 I5, Disponible en [Internet] http://www.retrovirology.com/content/6/I/98

38 García-Merino, Isabel et. al. Correspondence The Spanish HIV BioBank: a model of cooperative HIV research. Open Access Retrovirology 2009, 6:27, Búsqueda el 26 de agosto de 20 I5, Disponible en [Internet] http://www.retrovirology.com/content/6/I/27

39 García-Merino, Isabel et. al. Pediatric HIV BioBank: A New Role of the Spanish HIV BioBank in Pediatric HIV Research. AIDS Res Hum Retroviruses Volume 26, Number 2: 24I-4, 2010 Mary Ann Liebert, Inc. DOI: 10.1089=aid.2009. Búsqueda el 26 de agosto de 20 I5, Disponible en [Internet] http://online.liebertpub.com/doi/abs/I0.1089/aid.2009.0I22

40 Rosen, Rebecca J., Banks of Blood and Sperm, The Atlantic. Center for Genetics and Society, 31 de julio de 20I4, Búsqueda el 26 de agosto de 2015, Disponible en [Internet] http://www.geneticsandsociety.org/article.php?id=7952 
los biobancos, el acceso a la información debe ser garantizado, además de la "transferencia de tecnología, capacitación o beneficios para la población de estudio"4l Así los acuerdos previos suscritos, deben enmendarse para considerar este derecho y las modalidades de aplicación. Para que esto sea efectivo, la anonimización debe ser sustituida por una codificación (por ejemplo, la doble disociación aun cuando dificulta un potencial recontacto con el sujeto fuente) que preserve la confidencialidad y privacidad de los datos y muestras.

Entre los claroscuros que presenta el tema, nótese que la Declaración Universal sobre Bioética y Derechos Humanos sostiene que "la privacidad de las personas interesadas y la confidencialidad de la información que les atañe deberían respetarse. En la mayor medida posible, esa información no debería utilizarse o revelarse para fines distintos de los que determinaron su acopio o para los que se obtuvo el consentimiento" pudiendo producirse, como resultado del manejo discrecional de los datos, tanto un tratamiento imprudente o ilícito como un uso inapropiado.

\section{Situación en algunos países de América Latina}

La capacidad de responder a los desafíos del manejo ético de los biobancos, desde la privacidad y confidencialidad de los datos clínicos personales, es muy desigual entre los países de América Latina. ${ }^{42}$ No hemos encontrado leyes ni reglamentos específicos sobre biobancos en nuestros países. Sin embargo nuestras Constituciones y las leyes sobre derechos de pacientes aseguran la confidencialidad de los datos personales en los servicios de salud públicos y privados. No así la privacidad de las muestras biológicas, lo que constituye un problema jurídico al momento de defender los derechos de los donantes.

En Panamá, las políticas públicas están dirigidas a mantener un nivel suficiente de control epidemiológico y de satisfacción de las necesidades de la clase media que sostiene la economía de servicios y de comercio. En este marco, la regulación de los biobancos no constituye una prioridad legislativa. Las empresas que se establecen en el país aprovechan el vacío legal específico y obvian la Constitución y los acuerdos internacionales.

La profesión médica está dividida entre quienes consideran que los biobancos que operan actualmente usan publicidad engañosa sobre posibles curaciones y otros que se fascinan por lo que consideran tecnología de punta o eventualmente, por la participación económica que ello implica. La Ley 3 General de Trasplantes de Componentes Anatómicos, se refiere a "bancos de componentes anatómicos" en su capítulo II, para la "preparación, preservación, almacenamiento para tratamiento

4 Velosa, 20 10:135. Citado por Pinto Bustamente, B. et al - en: Bioética y la Brecha 10/90. Revista Redbioética/UNESCO, Año 5, 2 (I0): 87, julio - diciembre 20I4. ISSN 2077-9445. Búsqueda el 26 de agosto de 20I5, Disponible en [Internet] http://mww.unesco.org/new/fileadmin/MULTIMEDIA/FIELD/Montevideo/pdf/RevistaRBioet I 0-Final.pdf

42 Sotomayor Saavedra, María Angélica; López Dávila, Luis Manuel; Vergès, Claude; Sorokin, Patricia. Ficha clínica, protección de datos y derecho a la intimidad. Revista RedBioética/UNESCO, Año 5, 2 ( I 0): I I 9- I29, julio - diciembre 20 I 4 ISSN $2077-9445$. Búsqueda el 26 de agosto de 20 I5, Disponible en [Internet] http://www.unesco.org/new/fileadmin/MULTIMEDIA/FIELD/Montevideo/pdf/RevistaRBioet I 0-Final.pdf 
o investigación clínica debidamente documentada" (Art. 49) y fija los requisitos de exportación de células y tejidos hematopoyéticos (Art. 52) y de órganos anatómicos (Art. 18.2); a su vez el artículo 4 garantiza la confidencialidad de los datos del donante y el artículo 59 impone un "sistema único y obligatorio de codificación para identificar los tejidos y células" 43 Cultores de la Bioética y algunos grupos médicos han tratado de apoyarse en esta ley, así como en la Ley 17, particularmente el artículo 34 que prohíbe la exportación por organizaciones privadas, de la sangre, sus componentes y derivados. ${ }^{44}$

Sin embargo, el artículo 136 de la Ley 3 de trasplantes, establece que "Quedan excluidos de la ley la sangre o derivados así como los procedimientos de investigación in vitro de células que no incluyen una aplicación en el cuerpo humano", norma ésta que ha permitido a las diferentes compañías defender el derecho de operación de las mismas. Así operan Cordón de Vida, representante de Family Cord, Los Ángeles, Estados Unidos ${ }^{45}$ y CryoCell Panamá46. Ambas compañías se desenvuelven públicamente en el mercado nacional, con promoción en Internet y en las maternidades privadas que reciben usuarias nacionales y extranjeras de altos ingresos. Su calidad técnica no ha sido cuestionada pero sí su estrategia comercial que no considera en su integridad principios éticos tales como integridad, honestidad, precaución, responsabilidad y solidaridad. Anuncia sus productos y se ampara en un contrato con unos donantes voluntarios (personal o por substitución para los recién nacidos) que suscriben un contrato de seguro por una duración acordada (de un año a 25 años) y que respeta la confidencialidad de los datos al igual que un banco financiero y cumpliendo con la Ley 68 sobre Derechos de Pacientes ${ }^{47}$. Las muestras recolectadas son enviadas a la empresa madre en Estados Unidos, aunque abre la opción de un laboratorio en Panamá. Si bien ambas anuncian que las muestras pueden servir para investigación, la publicidad insiste sobre la curación de una lista larga de enfermedades mezclando las que responden a trasplantes hematopoyéticos con otras que están todavía bajo investigación en la literatura científica. Lo anterior colisiona con los principios y la mirada de la ética médica y los derechos humanos.

En Argentina, el Banco Nacional de Datos Genéticos (BNDG) es un organismo autónomo y autárquico, creado en el año 1987 por la Ley 235 I I. En 2009, tras ser sancionada la Ley 26548 pasó

43 Asamblea Nacional Ley 3 General de trasplantes de componentes anatómicos del 08-02-20 I 0 Gaceta Oficial:26468-b publicada el 10 de febrero de 2010 Búsqueda el 26 de agosto de 2015, Disponible en [Internet] http://www.asamblea.gob.pa

44 Asamblea Legislativa, República de Panamá Ley 17 por la cual se reglamentan los Bancos de Sangre en Panamá, 31 julio 1986. Publicada en la Gaceta Oficial 206I4 del 8 agosto 1986. Búsqueda el 26 de agosto de 20I5, Disponible en [Internet] http://200.46.254.138/legispan/PDF_NORMAS/I980/I986/I986_014_I617.PDF

45 Para ampliar información de los países donde se encuentra la empresa puede ver la página Web de Cordón de Vida Venezuela, 2009, Búsqueda el 26 de agosto de 2015, Disponible en [Internet] http://www.cordondevida.net.ve/site/web/precios_new. php?pais=2

46 CrioCell Panamá S.A. 20 I5. Búsqueda el 26 de agosto de 20 I5, Disponible en [Internet] http://www.cryocellpanama.com

47 Asamblea Nacional Ley 682003 Regula los derechos y obligaciones de los pacientes del 20 de noviembre 2003. Gaceta Oficial 24935 del 25 de noviembre 2003. Búsqueda el 26 de agosto de 2015, Disponible en [Internet] http://www.gorgas.gob.pa/wpcontent/uploads/2013/I I/Ley-68-2003-Regula-los-derechos-y-obligaciones-de-los-pacientes.pdf 
a funcionar bajo la jurisdicción del Ministerio de Ciencia, Tecnología e Innovación Productiva. ${ }^{48}$ Con respecto al BNDG se sabe que:

Es un archivo sistemático de material genético y muestras biológicas de familiares de personas que han sido secuestradas y desaparecidas durante la dictadura militar argentina. Su objetivo es garantizar la obtención, almacenamiento y análisis de la información genética que sea necesaria como prueba para el esclarecimiento de delitos de lesa humanidad y cuya ejecución se haya iniciado en el ámbito del Estado Nacional hasta el 10 de diciembre de 1983.49

Por su parte, en el Hospital de Pediatría Juan P. Garrahan funciona el Banco Público de Sangre de Cordón Umbilical. En el año 1996 se implementó allí un Programa orientado a "[.... ... familias que estuvieran esperando un niño y ya tuvieran otro hijo con una enfermedad tratable con trasplante de médula ósea." 50 En 2005 comenzaron las actividades destinadas a la "[... . . . Colecta de Sangre de Cordón Umbilical en el cual las familias son invitadas a donar en forma altruista la sangre de cordón de su niño. Ésta pasa a formar parte del Banco Público y queda a disposición de la población de pacientes que requieren un transplante de médula ósea y no cuentan con un donante relacionado." 51

La situación en Chile, muestra ausencia de regulación específica sobre biobancos y no está prohibida la exportación de material biológico. La Ley sobre Investigación No 20.120 uno de cuyos objetivos es la protección de la diversidad e identidad genética de las personas en relación con la investigación científica biomédica y sus aplicaciones clínicas, permite la terapia génica sólo con fines de tratamiento y prevención de enfermedades. Prohíbe la destrucción de embriones para obtener las células troncales que den origen a dichos tejidos y órganos. Para la investigación y determinación genética de un ser humano exige consentimiento informado y califica la información genética de un ser humano como reservada, aplicando a su respecto normas sobre secreto profesional. Aplica las normas sobre protección de datos de carácter personal (Ley $N^{0}$ 19.628) ${ }^{52}$ a la recopilación, almacenamiento, tratamiento y difusión del genoma de las personas, obligando a que los datos del genoma humano que permitan la identificación de una persona, sean encriptados para su almacenamiento y transmisión, la que puede omitirse temporalmente por razones de utilidad pública. Finalmente, prohíbe solicitar, recibir, indagar, poseer y utilizar información sobre el genoma relativo a una persona, salvo que ella lo autorice expresamente o, en su defecto el que deba suplir su voluntad en conformidad con la ley. Excluye de esta norma a la información

48 Presidencia de la Nación, Ministerio de Ciencia, Tecnología e Innovación Productiva, Banco Nacional de Datos Genéticos (BNDG), Búsqueda el 30 de agosto de 20 I5, Disponible en [Internet] http://www.mincyt.gob.ar/ministerio/banco-nacional-dedatos-geneticos-bndg-23

49 Ídem.

50 Hospital de Pediatría Prof. Dr. Juan P. Garrahan, Banco Público de Sangre de Cordón Umbilical. Búsqueda el 30 de agosto de 20I5, Disponible en [Internet] http://www.garrahan.gov.ar/nosotrosdonamos/quienes-somos-BSCU.php

51 Ídem.

52 Congreso Nacional de Chile, Ley N 19.628 de 1999 sobre "Protección de la Vida Privada" denominada también "Sobre protección de datos de carácter personal" Búsqueda el 29 de agosto de 20I5, Disponible en [Internet] http://www.leychile. $\mathrm{cl} / \mathrm{N} ? \mathrm{i}=\mid$ | | $599 \& \mathrm{f}=20$ | 2-02- 17 \&p 
con fines forenses. En su caso, el reglamento de esta ley ${ }^{53}$ viene a complementar sus normas en cuanto a que el cultivo de tejidos y órganos con fines diagnósticos, terapéuticos o de investigación científica, debe realizarse en laboratorios clínicos o de salud pública autorizados. Obliga a quienes conserven muestras o elementos biológicos con fines de investigación, diagnóstico o tratamiento, a "adoptar las medidas necesarias para su seguridad y confidencialidad que sean suficientes para resguardar su correcto uso, debiendo destinarlos exclusivamente a los fines para los cuales fueron obtenidos" 54 . Por su parte la Ley sobre Derechos y Deberes de los Pacientes ${ }^{55}$ restringe el acceso de terceros a la historia o ficha clínica del paciente y hace aplicable a las muestras biológicas la Ley sobre Protección de la Vida Privada, que regula el acceso a datos de carácter personal y dentro de éstos a los datos sensibles de una persona. En conclusión a este respecto, si bien Chile cuenta con regulación protectora de la privacidad, no ha regulado específicamente a los biobancos. El país participó junto a Alemania, Argentina, Brasil, Colombia, Costa Rica, España, Francia, México y Portugal en el proyecto LATINBANKS que se desarrolló en el marco del programa ALFA de la Unión Europea, y que se propuso precisamente recopilar información sobre los bancos de materiales (muestras) biológicos humanos para investigación in vitro, como parte de las actividades de la red MEDNET conformada por las instituciones participantes, casi todas ellas universidades, dedicada al estudio de los problemas jurídicos de los avances en biomedicina. ${ }^{56}$ En cuanto biobancos, el Servicio Médico Legal ${ }^{57}$ implementa el "Sistema Nacional de Registro de ADN" constituido sobre la base de huellas genéticas de la población a través del análisis de muestras, registro y custodia de éstas, para la resolución de casos criminales y búsqueda de personas perdidas. En el área privada se observa una oferta similar a la de los países vecinos respecto de los bancos de cordón umbilical, en general recolectados en maternidades de clínicas privadas. Existen diversas iniciativas en desarrollo respecto de biobancos específicos, entre éstos en el área oncológica.

En el contexto de Guatemala no existen biobancos. Sin embargo con el advenimiento de la investigación biomédica internacional que recluta sujetos de experimentación en el país, con el uso de protocolos de ensayos clínicos multicéntricos se solicitan muestras para biobancos. Al no existir en el país regulación sobre el tema, en general los protocolos indican que las muestras serán enviadas a otro país. En cuanto a los aspectos de regulación que se observan se encuentran las desarrolladas para el manejo y traslado de muestras biológica según las regulaciones de la Asociación Internacional de Transporte Aéreo (IATA por sus siglas en inglés) ${ }^{58}$.

53 Ministerio de Salud de Chile, Decreto Supremo N ${ }^{\circ}$ I I 4 de 2010 Reglamento de la Ley 20 I 20 sobre Investigación Científica en el Ser Humano. Búsqueda el 28 de agosto de 2015, Disponible en [Internet] http://www.leychile.cl/Navegar?idNorma=1032919

54 Ídem.

55 Congreso Nacional de Chile, Ley No 20.584 de 2012 que Regula los Derechos y Deberes que tienen las Personas en relación con acciones vinculadas a su atención de Salud. Arts. 12 y. 13. Búsqueda el 29 de agosto de 20 I 5, Disponible en [Internet] http:// www-leychile.cl/N?i= | 03934\&f=20 I 2- I0-0 I \&p

56 Proyecto LATINBANKS, Búsqueda el 27 de agosto de 2015, Disponible en [Internet] http://www.latinbanks.catedra derechoygenomahumano.es/

57 Ministerio de Justicia, Gobierno de Chile, Servicio Médico Legal, Búsqueda el 29 de agosto de 20I5, Disponible en [Internet] http://www.sml.cl/sml/index.php?option=com content\&view=article\&id=70\&ltemid=156

58 Para ampliar información sobre la IATA se puede visitar el Sitio Web de la IATA, 20I5. Búsqueda el 3 I de Agosto de 20 I5. Disponible en [Internet] http://www.iata.org/whatwedo/pages/index.aspx 
Para la obtención de las muestras se presenta un consentimiento o asentimiento informado, donde se indica que serán manejadas con un código que no identifica directamente al paciente y que en el biobanco serán doblemente codificadas, quedando a cargo de personal autorizado el resguardo del primer código que tiene vínculo directo con los datos del paciente, por si es necesario llegar a éste según los resultados de los análisis o bien si el paciente solicita la destrucción de sus muestras. La regulación actual contenida en el Acuerdo Ministerial SP-M-466-2007 del Ministerio de Salud Pública y Asistencia Socia ${ }^{59}$ para la Regulación de los Ensayos Clínicos en Humanos, no contempla los aspectos para el manejo de muestras fuera de las fronteras, lo que se traduce en una ausencia de jurisprudencia. Los marcos regulatorios que se aplican son aquellos bajo la jurisdicción donde operan los biobancos.

El escenario anterior advierte la debilidad que tiene la protección del manejo de datos en cuanto a las garantías de confidencialidad y uso de las muestras. Aun cuando existen leyes que se aplican para los datos sensibles en el contexto de Guatemala como el Decreto número 57-2008 del Congreso de la República que contiene la Ley de Acceso a la Información Pública, el artículo 9 en la definición 2 aborda los "datos sensibles o datos personales sensibles". Sin embargo, el capítulo quinto sobre información confidencial y reservada y lo concerniente al capítulo sexto de Habeas Data ${ }^{60}$, ninguno de estos recursos establece regulación sobre el manejo de datos privados relacionados con investigación biomédica internacional. Al recabar información en una Organización de Investigación por Contrato (CRO por sus siglas en inglés) sobre los procedimientos que se desarrollan en el país con respecto a los biobancos, se indica que los criterios son establecidos por encargo del cliente (es decir el patrocinador de la industria farmacéutica) bajo estándares internacionales de calidad que éste establece y los certificados internacionales.

El contexto antes descrito muestra la importancia de la relación del modelo económico capitalista en el cual se regulan las garantías de mercado, en cuanto al manejo y traslado de muestras, dado que la debilidad de orden jurídico no protege la condición del propietario de la muestra. Se está frente a un escenario de uso de las condiciones de vulnerabilidad, que no permiten la protección o garantías futuras para revertir en los sujetos de experimentación, beneficios potenciales que sucederían con la aplicación de biotecnología. Se ha pasado de un esquema de utilización de sujetos de experimentación de las fases de investigación clínica-farmacológica a un esquema de uso potencial genético de las muestras que devienen de los sujetos de experimentación.

Los países de la región que carecen de marcos regulatorios y acceso a la biotecnología tienen una brecha de desigualdad que se incrementa con la falta de políticas de salud pública orientadas a la resolución de investigación autóctona donde los biobancos pueden ser un asidero local para los problemas de salud prioritarios de países de bajos ingresos. Esta condición de brecha de desigualdad

59 Ver Acuerdo Ministerial SP-M-466-2007, en sitio Web del Ministerio de Salud Pública y Asistencia Social de la República de Guatemala, 0 I de Febrero de 2007. Búsqueda el 3 I de Agosto de 20 I5. Disponible en [Internet] http://www.mspas.gob.gt/files/ Descargas/DGRVCS/Salud_publica/Acuerdos\%204/AMSPM46607.pdf

60 Congreso de la República de Guatemala, Decreto Número 57-2008, sitio Web del Instituto Nacional de Estadística Guatemala, 2013, Búsqueda el 3I de Agosto de 20I5. Disponible en [Internet] http://www.ine.gob.gt/archivos/informacionpublica/ LeydeAccesoalalnformacionPublica.pdf 
aumenta en tanto los biobancos están fuera de las fronteras y lejos de dar una respuesta a necesidades locales, dado que el interés fundamental por el cual se han recolectado las muestras en general, atiende a problemas de salud foráneos y a la agenda económica de la industria farmacéutica internacional.

\section{Conclusión}

Se torna imperioso generar conciencia respecto de los desarrollos científicos e informáticos ya que éstos desdibujan las fronteras y consecuentemente se torna necesario adoptar tanto a nivel individual como conjunto, las medidas necesarias para preservar los derechos de sus habitantes.

En perspectiva de la "Democratización" de los biobancos se debe apuntalar en el respeto a la privacidad, el cual es un elemento esencial de la programación de los biobancos en un país o grupo de países. De este respeto se establecerán las modalidades de confidencialidad, acceso y revocación de consentimiento por parte de los donantes y se ganará la confianza de la ciudadanía. Para ello es necesario reforzar la capacidad de los comités de bioética existentes y fomentar su creación local y nacional. Pero también es necesario consultar a la ciudadanía sobre su percepción de los biobancos y su anuencia a participar en ellos en un marco de respeto de derechos de la generación actual y futura.

La consideración de una institucionalidad respetuosa de los derechos humanos y de sensibilidad, cultura étnica de la población y biodiversidad de la Región, organizada sobre la base de un acuerdo internacional en el marco del Pacto de San José de Costa Rica, podría facilitar la confianza de la comunidad de América Latina, para optar a los beneficios de la ciencia y de la asistencia sanitaria, en biobancos de amplio acceso, bajo el alero de seguridad y respeto irrestricto de los derechos humanos y de respeto por las personas. La creación y fortalecimiento de Comités de Ética que afronten este desafío y que respondan a criterios éticos, de probidad, calidad e independencia, resulta indispensable.

La solidaridad asume que la atomización de un colectivo hace más vulnerable a cada uno de sus miembros, por lo cual es recomendable entender este colectivo como un sólido (= solidaridad) que aúna los esfuerzos de todos para paliar infortunios, defenderse de agresiones, constituir un sistema de protección y elaborar en conjunto para la obtención de bienes comunes. Implícita en la solidaridad está la igualdad, tanto de esfuerzo realizado como de protección obtenida, y allí donde esta simetría se rompe, aparecen los abusos y las desprotecciones de los más débiles.

\section{Bibliografía}

Anguita, V. y Sotomayor, M. A. "¿Confidencialidad, anonimato?: las otras promesas de la investigación”. Acta Bioethica. 17(2) 201 I: 199-204.

Austin, M.; Harding, S. y McElroy, C. Genebanks: a Comparison of Eight Proposed International Genetic Databases. Community Genet. 6(I), (2003): 37-45. 
Barbour, V. “UK Biobank: a project in search of a protocol?"' Lancet. 36I (9370), (2003): I 734-1738.

Bergel, S., Sorokin, P., Justo, L.; Torres, S.; Pagano, L.; Ghioldi. C. y del Percio, D. "Confidencialidad y Genoma Humano: hacia una indispensable propuesta legislativa" En BIO-PHRONESIS. Revista de Bioética y Socioantropología en Medicina. Búsqueda el 26 de agosto de 20 I5. Disponible en [Internet] http://fmv-uba.org.ar/comunidad/ revistasylibrosdigitales/antropologia/Nro IMarzo2006/sorokin.asp

Bovenberg, J.; Meulenkamp, T.; Smets, E. y Gevers, S. Biobank research: Reporting Results to Individual Participants. European Journal of Health Law. 16(3), (2009); 229-247.

Cambon-Thomsen, A.; Ducournau, P.; Gourraud, P-A. y Pontille, D. Biobanks for Genomics and Genomics for Biobanks. Comparative and Functional Genomics. 4(6), (2003): 628-634.

Cambon-Thomsen, A. "The social and ethical issues of post-genomic human biobanks". Nature (5), 2004: 867.

Cambon-Thomsen, A.; Rial-Sebbag, E. y Knoppers, B. Trends in Ethical and Legal Frameworks for the Use of Human Biobanks. European Respiratory Journal (30), (2007): 373-382.

Cecchetto, S. La biología contra la democracia. Eugenesia, herencia y prejuicio en Argentina. 1880- 1940. Mar del Plata: EUDEM, 2008.

Elger, B. y Caplan, A. Consent and Anonymization in Research Involving Biobanks, EMBO reports 2006 7(7): 66 I -666.

Kaiser, Jocelyn. Biobanks. Population databases boom, from Iceland to the U.S. Science. 298(5596), (2002): I I581161.

Keyeux, G. Bancos de datos genéticos humanos: el equilibrio vacilante entre ciencia y derechos humanos. En V. Penchaszadeh (comp.) Genética y Derechos Humanos: Encuentros y Desencuentros. Buenos Aires: Biblioteca Nacional-Paidós, 20 I2: 237-262.

Minott, C.; Moreno, M. del R.; Scrigni, A. y Dacoll, C. "Protección de datos sensibles: un desafío". Número Especial de Jurisprudencia Argentina sobre Derechos personalísimos y VIH/sida en el nuevo decenio. P. Sorokin (coord.) Buenos Aires: AbeledoPerrot, I(8), (2011): 92-96.

Moreno, M. del R. y Pinto, A. L. "El consentimiento informado en el marco de las investigaciones clínicas y la aplicación de normas de confidencialidad" En P. Sorokin (coord.) Número Especial de Jurisprudencia Argentina sobre Derechos personalísimos, VIH/sida y prensa escrita. Buenos Aires: LexisNexis, I(8), (2008): 79-82.

Pfeiffer, M. L. "Derecho a la privacidad. Protección de datos sensibles". Revista Colombiana de Bioética. Colombia: Universidad El Bosque, 2008: II-36.

República Argentina. Ministerio de Salud. Secretaria de Políticas, Regulación e Institutos ANMAT Disposición 6677 (20 I0) Búsqueda el 26 de agosto de 20 I5, Disponible en [Internet] http://www.anmat.gov.ar/Comunicados/ Dispo_6677-10.pdf

República Argentina. Ley № 25.326 de Protección de los Datos Personales (2000). Búsqueda el 26 de agosto de 20I5, Disponible en [Internet] http://www.infoleg.gov.ar/infoleglnternet/anexos/60000-64999/64790/ norma.htm 
República Argentina. Ley 26.529. Derechos del Paciente en su Relación con los Profesionales e Instituciones de la Salud (2009). Búsqueda el 26 de agosto de 2015, Disponible en [Internet] http://www.infoleg.gov.ar/ infoleglnternet/anexos/160000-164999/160432/norma.htm

Sorokin, P. Judicial Perceptions of Sensitive Information in Buenos Aires. International Conference on Ethical, Legal and Social Aspects of Human Genetic Databases. University of Iceland in Reykjavik. Iceland: 25-28/08/2004.

Sorokin, P. "Tratamiento de datos personales en investigación genómica: aspectos éticos, legales y sociales" en F. Lolas Stepke y J. G de Freitas Drumond (dirs.) Bioética. Editorial Mediterráneo. Santiago: 20I3, pp. 307-3I9. ISBN: 978-956-220-394-4.

Wendler, D. y Emanuel, E. The Debate Over Research on Stored Biological Samples: What do Sources Think? Archives of Internal Medicine, I62(13), (2002): | 457- | 462. 